\title{
Modelling of the joint motion of nonisothermal liquid film and gas flow in a microchannel: numerical simulation of full Navier-Stokes equations
}

\author{
Vladimir V. Kuznetsov ${ }^{1}$ and Yulia O. Kabova ${ }^{2, a}$ \\ ${ }^{1}$ Lavrentyev Institute of Hydrodynamics, SB RAS, 630090, 15 Lavrentyev prosp., Novosibirsk, Russia \\ ${ }^{2}$ Institute of Thermophysics, SB RAS, 630090, 1 Lavrentyev prosp., Novosibirsk, Russia
}

\begin{abstract}
New full-statement 3D mathematical model of joint motion of thin liquid film and gas in a microchannel at local heating developed by taking into account the heat transfer by flows, evaporation and condensation, as well as the heat transfer at the gas-liquid interface is derived. The model is based on the full system of the Navier-Stokes equations, taking into account the convective terms of motion equations in the phases. Comparison of the numerical results obtained using the model based on the full Navier-Stokes equations and using the simplified model developed in the framework of the thin layer approximation has been performed. The comparison shows that at low Reynolds numbers, simplified model well describes all the main characteristics of the gas and liquid motion. With the gas Reynolds numbers significant increase difference between numerical results starts to grow.
\end{abstract}

\section{Introduction}

Flows of thin liquid films driven by various forces such as gas flow, gravity, capillarity, thermocapillarity and intermolecular forces are encountered in cooling devices, heat-exchangers, microfluidic devices, in wetting and spreading, in condensers, as well as in biomedical and geophysical applications. Over the past decades, numerous theoretical and experimental research works have been focused on the dynamics and heat transfer in thin liquid films, evaporation and condensation processes were taken into account in many studies $[1,2,3,4,5]$. Particularly, big variety of mathematical models taking into account different features of the liquid motion and heating has been developed using the lubrication theory $[6,7,8]$, and also numerical simulation of the full NavierStokes equations under certain conditions [9] has been performed. Method of the integral proportions proposed by Prof. V.Ya. Shkadov also gives good results [10], but the question about impact of the gas phase on the free surface phenomena remains poorly studied. Generally, investigation of joint influence of thermocapillary and mass forces is conducted within the framework of generally accepted models, while full understanding of mechanisms of the structures formation and of influence of heat and mass transfer effects requires the use of new and more complex mathematical models taking into account the convective terms of equations. The first model of joint liquid and gas motion taking into account convective heat transfer in liquid and gas phases, as well as evaporation, which has been

a Corresponding author: kabova@itp.nsc.ru 
described by the convective diffusion equation in the gas phase has been proposed in [11] for laminar stratify flow with nondeformable free interface. In $[12,13]$ a 3D simulation of evaporating liquid and gas joint motion in a microchannel at local heating is presented. The hydrodynamics has been described in the framework of the lubrication approximation, whereas convective heat transport in both phases and convective diffusive vapor transport in the gas phase have been taken into account. It should be noted that full statement three-dimensional models, describing the liquid films motion taking into account the gas phase and the phase changes at the free boundaries are still very rare in literature. In the present work new full statement three-dimensional mathematical model of joint motion of a viscous liquid film and gas in a microchannel at local heating developed by taking into the inertial terms in motion equations as well as convective heat transport in both phases and convective diffusive vapor transport in the gas phase. Gas compressibility is neglected.

\section{Problem statement}

Consider a thin film of incompressible viscous liquid sheared by gas inside a microchannel of given height $\mathrm{H}_{\mathrm{C}}$. The microchannel is assumed to be unbounded in $x$ and $y$ directions. A part of wall at the side of liquid film is heated (Fig. 1). The transport processes in the liquid and in the gas are described by the Navier-Stokes, continuity and energy equations, as well as by diffusion equation in the gas phase.

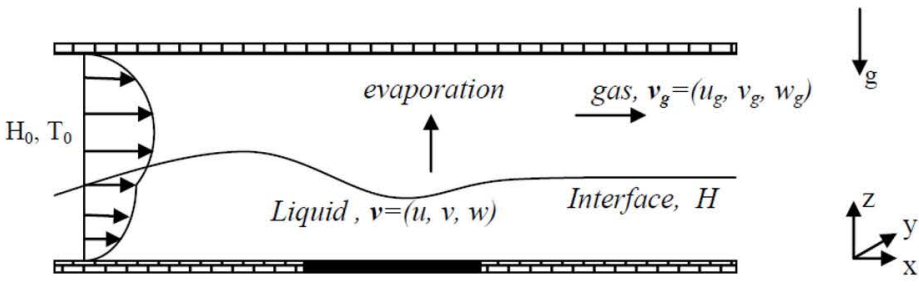

heater

Figure 1. A sketch of the physical model.

The initial film thickness, tangential stresses, the pressure drop, and velocity fields in the liquid and gas phases are determined from the exact solution of the problem of isothermal laminar co-current flow in the channel with straight streamlines. The upper wall is assumed to be adiabatic and impermeable. At the bottom wall no slip condition and thermal boundary conditions, prescribing the constant heat flux, $\mathrm{q}$, or constant temperature distribution, $\mathrm{T}_{1}$, at the heater, are posed. At the gasliquid interface the condition of continuity of the tangential component of the velocity vector (no-slip condition of two viscous media), mass conservation for the fluid comprising evaporating film and mass conservation conditions, dynamic condition with the term expressing the mechanical effect of the evaporated matter on the liquid, thermal boundary condition, taking into account the energy spending on the phase transition, as well as condition of local thermodynamic equilibrium are posed. The concentration, surface tension and dynamic viscosity are assumed to be temperature dependent. All details with regard to the problem statement could be found in $[11,12]$ for the case of the lubrication approximation.

\section{Development of the mathematical model}

In the [11] the lubrication theory has been employed to derive reduced set of equations, so the film aspect ratio has been taken asymptotically small $\varepsilon=H_{0} / l<<1$. And considering inertial terms to be negligible, the system of equations with boundary conditions has been rewritten in dimensionless form. And afterwards has been reduced to a solution of five governing equations: for the film thickness, temperature distribution in liquid and gas, vapor concentration in the gas and gas pressure. 
Let us assume here that inertial terms in the motion equations are not negligible and should be taken into account. To derive the full statement mathematical model, let us perform the change of variables in the liquid and gas phases as follows:

$$
\begin{aligned}
& \xi=z / H, u^{1}=u H, v^{1}=v H, w^{1}=w-u \xi H_{x}-v \xi H_{y}, \\
& \eta=\frac{\omega-1}{H_{C}-H} z-\frac{H \omega-H_{C}}{H_{C}-H}, \quad u_{g}^{1}=\frac{H_{C}-H}{\omega-1} u_{g}, \\
& v_{g}^{1}=\frac{H_{C}-H}{\omega-1} v_{g}, w_{g}^{1}=w_{g}-\frac{H_{C}-z}{H_{C}-H} H_{x} u_{g}-\frac{H_{C}-z}{H_{C}-H} H_{y} v_{g}
\end{aligned}
$$

Where $\omega=H_{C} / H_{0}$. This change of variables leads to simplification of the problem: spatial variables become nondependent on time and having flat free border and the kinematic interfacial conditions is simplified and becomes linear. Then the governing equations with boundary conditions could be rewritten in the layer $\xi \in(0,1), \eta \in(1, \omega),-\infty<x, y<\infty$. The continuity equations in the liquid and in the gas retain their form in new variables but kinematic condition at the free interface simplifies. To rewrite governing equations with boundary conditions in the dimensionless form let us define scales for the liquid velocity, longitudinal length and temperature as follows:

$$
U=\frac{\mu_{0}}{\rho H_{0}}, l=\left(\frac{\sigma_{0} H_{0}^{2}}{\rho U^{2}}\right)^{1 / 3}, \mu_{0}=\mu\left(T_{0}\right),[T]=\frac{Q_{0} H_{0}}{\kappa S_{0}} \text { or }[T]=\underset{t, x, y}{\max }\left(T_{1}(t, x, y)-T_{0}\right) .
$$

Here $\mathrm{Q}_{0} / \mathrm{S}_{0}$ is the average heat flux at the heater, $\mathrm{S}_{0}$ the heating area. System of equations with boundary conditions has been rewritten in dimensionless form with the following dimensionless criteria of similarity and coefficients:

$$
\begin{aligned}
& \mathbf{A}=\frac{g \cos \alpha H_{0}^{2}}{U^{2} l}, \quad \mathbf{C}=\frac{g \sin \alpha H_{0}}{U^{2}}, \quad \mathbf{M a}=\frac{\sigma_{T}[T] H_{0}}{\mu_{0} U l}, \mathbf{L}=\frac{\lambda \rho_{g} D}{\kappa[T]}, \\
& \mathbf{S h}=\frac{\mu_{g}}{\rho_{g} D}, \mathbf{C r}=\frac{\sigma_{T}[T]}{\sigma_{0}}, \mathbf{C a}^{-1}=\frac{\mu_{0} U l^{2}}{\sigma_{0} H_{0}^{2}}, \mathbf{R}=\frac{D l}{H_{0}^{2} U}, \quad \mathbf{P r}=\frac{c_{p} \mu_{0}}{\kappa}, \\
& \mathbf{P r}_{\mathbf{g}}=\frac{c_{p g} \mu_{g 0}}{\kappa_{g}}, \quad \mathbf{P}=\frac{\rho_{g} D l^{2}}{\sigma_{0} H_{0}^{3}}, k_{1}=\frac{\mu_{0 g}}{\mu_{0}}, k_{2}=\frac{\kappa_{g}}{\kappa}, k_{3}=\frac{\rho_{g}}{\rho} .
\end{aligned}
$$

Where $\alpha$ - channel inclination angle, $\kappa$ - thermal conductivity , $\lambda$ - latent heat of vaporization, $\mu$ liquid dynamic viscosity, $\rho$ - liquid density, $\sigma$ - surface tension, $D$ - diffusion coefficient, $c_{p}$ specific heat of the liquid. Afterwards dimensionless system of equations with boundary conditions has been reduced to a solution of five governing equations: for the film thickness, temperature distribution in liquid and gas, vapor concentration in gas phase and gas pressure. Numerical solution of the problem is implemented by the finite difference method. The alternating directions implicit (ADI) method is used to solve the system of grid equations with boundary conditions. To solve the problem on each fractional grid step the Thomas algorithm is used. Channel height average pressure in liquid and gas has been calculated using the following formulas:

$$
\tilde{p}(x, y, t)=\int_{0}^{1} p(x, y, \xi, t) d \xi, \quad \tilde{p}_{g}(x, y, t)=(1 /(\omega-1)) \int_{1}^{\omega} p_{g}(x, y, \xi, t) d \xi .
$$

This leads to a simplification of the numerical solution of the problem. Material constants correspond to water and nitrogen, inclination angle is equal to $0^{\circ}[14]$.

\section{Results}

Calculations have been performed for the constant channel height $\mathrm{H}_{\mathrm{C}}=500 \mu \mathrm{m}, \alpha=0$, heater size is equal to $3 \times 6 \mathrm{~mm}^{2}$ (length $\times$ width) and the heat flux $\mathrm{q}=0.5 \mathrm{~W} / \mathrm{cm}^{2}$. The initial temperature is equal 
to $30^{\circ} \mathrm{C}$. Nondimensional evaporation rate from the gas-liquid interface, $\mathbf{J}_{\text {total }}$, is calculated using formula $\mathbf{J}_{\text {total }}=\left.\int_{\Lambda} \frac{1}{h(x, y)} \frac{\partial C}{\partial \xi}\right|_{\xi=1} d x d y$, here $\Lambda$ is the area of calculations, $C$ - mass fraction of moisture in the gas phase. $\mathbf{J}_{\text {total }}$ is proportional by physical meaning to the value $\left(C_{0} D \rho_{g} l^{2}\right) / H_{0}[\mathrm{~kg} / \mathrm{s}]$. Reynolds numbers $\mathbf{R e}=Q \rho / \mu_{0}, \mathbf{R e}_{g}=Q_{g} \rho_{g} / \mu_{0 g}$ characterize flow intensity in the liquid and gas, here $Q, Q_{g}$ - fluid and gas flow rates per unit width, correspondingly. All values presented in the graphs are non-dimensional, namely $t=\hat{t} l / U, T=T_{0}+[T] \hat{T}, H=H_{0} h$. Influence of the liquid and gas Reynolds numbers values on the main process characteristics including the interface deformations, maximum temperature and evaporation has been investigated numerically. Reynolds numbers in the liquid and gas are varied from 2 to 25 and from 15 to 200, correspondingly, Fig. 2-3.

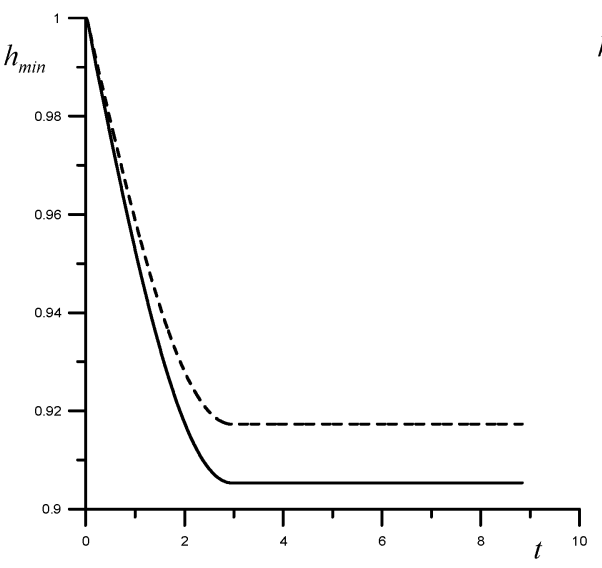

(1)

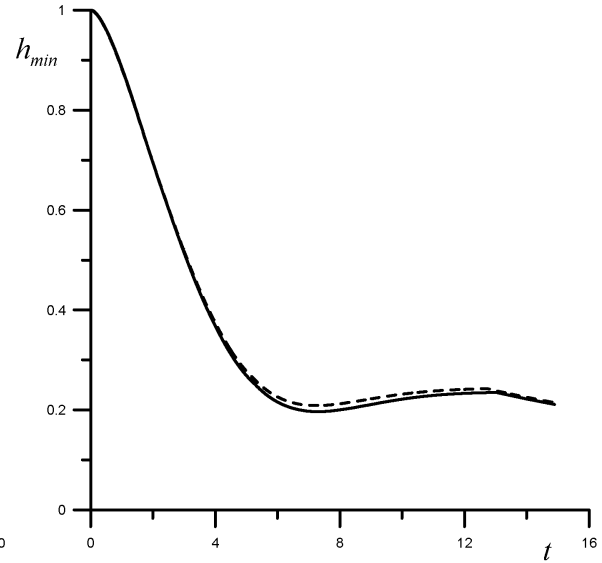

(2)

Figure 2. Minimum film thickness versus time. Dashed lines - full Navier-Stokes equations, solid lines lubrication approximation. (1) $-\mathrm{Re}=25$ and $\mathrm{Re}_{\mathrm{g}}=200$; (2) $-\mathrm{Re}=2,5$ and $\mathrm{Re}_{\mathrm{g}}=20$.

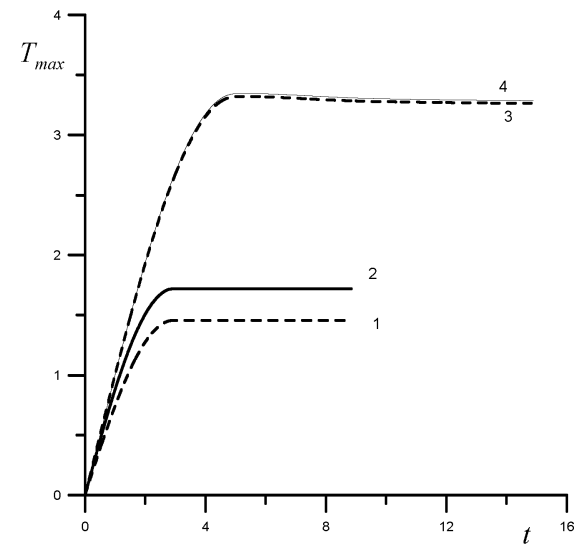

(1)

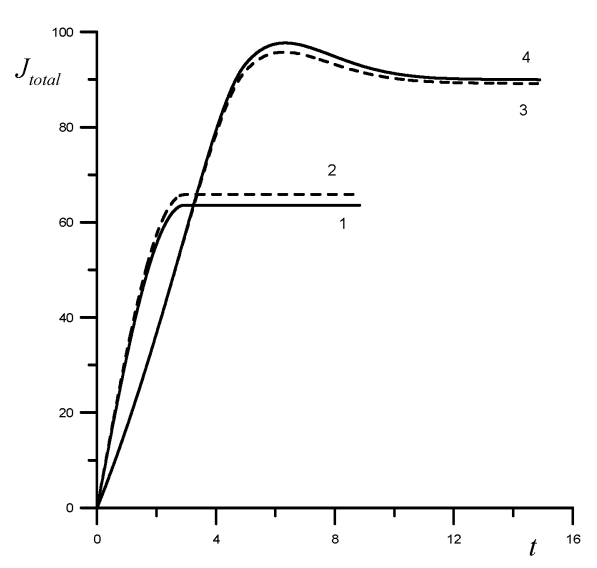

(2)

Figure 3. (1) - Maximum dimensionless temperature at the gas -liquid interface. (2) - Evaporation rate from the gas-liquid interface. Dashed lines - full Navier-Stokes equations, solid lines - lubrication approximation. (1) - Re $=25$ and $\operatorname{Re}_{\mathrm{g}}=200 ;(2)-\operatorname{Re}=2,5$ and $\operatorname{Re}_{\mathrm{g}}=20$. 
In addition, comparison of the numerical results obtained using the model based on the full NavierStokes equations and using the simplified model developed in the framework of the thin layer approximation has been performed. The comparison shows that at low values of Reynolds numbers, simplified model well describes all the main characteristics of the gas and liquid motion. As one can see in Fig. 2 and 3 the difference between the calculated parameters for both models does not exceed $4 \%$. Lines 1 and 2 correspond to the Reynolds numbers $R e=25$ and $\operatorname{Re}_{\mathrm{g}}=200$ as well as lines 3 and 4 to the $\mathrm{Re}=2,5$ and $\mathrm{Re}_{\mathrm{g}}=20$. With the gas Reynolds number significant increase differences between numerical results starts to grow.

\section{Conclusion}

New full statement three-dimensional mathematical model of joint motion of a viscous liquid film and gas in a microchannel at local heating developed by taking into account the heat transfer by flows, evaporation and heat transfer at the gas-liquid interface is derived. The model is based on the full system of Navier-Stokes equations, taking into account the inertial terms in motion equations. Comparison of the numerical results obtained using the model based on the full Navier-Stokes equations and using the simplified model developed in the framework of the thin layer approximation is performed. The comparison shows that at low Reynolds numbers, simplified model well describes all the main characteristics of the gas and liquid motion. With the gas Reynolds numbers significant increase difference between numerical results starts to grow.

\section{Acknowledgements}

The study was financially supported by the Russian Science Foundation, project no. 14_19_01755.

\section{References}

1. J. P. Burelbach, S.G. Bankoff, S. H. Davis, Journal of Fluid Mechanics, 195 (1988)

2. R.V. Craster, O.K. Matar, Reviews of Modern Physics, 81 (2009)

3. E.A. Chinnov and O.A. Kabov, Microgravity Science and Technology, XIX-3/4 (2007)

4. O.A. Kabov, Yu.V. Lyulin, I.V. Marchuk and D.V. Zaitsev, International Journal of Heat and Fluid Flow, 28, 103 (2007)

5. D.V. Zaitsev, D.A. Rodionov and O.A. Kabov, Technical Physics letters, 35, 680 (2009)

6. A. Oron, S. H. Davis, S. G. Bankoff, Reviews of Modern Physics, 69 (1997)

7. J.M. Skotheim, U. Thiele, B. Scheid, Journal of Fluid Mechanics, 475 (2003)

8. V.S. Ajaev, E.Ya. Gatapova, O.A. Kabov, Physical Review E, 84, 4 (2011)

9. A.M.Frank, O. A. Kabov, Physics of Fluids, 18 (2006)

10. S. Kalliadasis, A. Kiyashko and E.A. Demekhin, Journal of Fluid Mechanics, 475 (2003)

11. E.Ya. Gatapova, O.A. Kabov, International Journal of Heat and Mass Transfer, 51, 19-20 (2008)

12. Yu. Kabova, V.V. Kuznetsov, O. Kabov, T. Gambaryan-Roisman, P. Stephan, International Journal of Heat and Mass Transfer 68 (2014)

13. Yu. Kabova, V.V. Kuznetsov, O. Kabov, Interfacial Phenomena and Heat Transfer 2,1 (2014)

14. Tables of physical units. Handbook. (Ed.: Acad. I.K. Kikoin. M.: Atomizdat, 1976) 